\title{
Mesh shrinkage and pain in laparoscopic ventral hernia repair: a randomized clinical trial comparing suture versus tack mesh fixation
}

\author{
Guido Beldi • Markus Wagner • Lukas E. Bruegger • \\ Anita Kurmann • Daniel Candinas
}

Received: 3 March 2010/ Accepted: 8 July 2010/Published online: 23 July 2010

(C) Springer Science+Business Media, LLC 2010

\begin{abstract}
Background Mesh fixation during laparoscopic ventral hernia repair can be performed using transfascial sutures or metal tacks. The aim of the present study is to compare mesh shrinkage and pain between two different techniques of mesh fixation in a prospective randomized trial.

Methods A randomized trial was performed. Patients with ventral hernia of maximal diameter $8 \mathrm{~cm}$ were assigned to mesh fixation using either transfascial nonabsorbable sutures or metal tacks for fixation of a parietene composite mesh. The borders of the mesh were marked using clips, and radiological images in prone position were used for assessment of mesh size and location. The primary endpoint was mesh shrinkage; secondary endpoints included postoperative pain, mesh dislocation, and surgical morbidity.

Results Demographic parameters were similar in both groups. A total of 40 patients were randomized, and 36 patients were available for follow-up. There was one hernia recurrence in each group. Pain was significantly higher following suture fixation after 6 weeks, but no difference was found after 6 months. Mesh shrinkage after 6 months was significantly higher using tacks for mesh fixation.

Conclusions Transfascial sutures are associated with more pain within the first 6 postoperative weeks and less mesh shrinkage after 6 months compared with mesh fixation using metal tacks.
\end{abstract}

Guido Beldi, Markus Wagner contributed equally to this work.

G. Beldi $(\bowtie) \cdot$ M. Wagner $\cdot$ L. E. Bruegger · A. Kurmann D. Candinas

Department of Visceral Surgery and Medicine, Bern University Hospital, University of Bern, 3010 Bern, Switzerland

e-mail: guido.beldi@insel.ch
Keywords Incisional hernia - Laparoscopy · Mesh fixation · Pain · Recurrence

Laparoscopic ventral hernia repair (LVHR) has been shown to be safe and feasible [1-4]. In comparison with open techniques, LVHR is associated with reduced incidence of surgical-site infections and reduced hospital stay at comparable costs [1, 5-8]. However, in most of these studies, pain was not significantly reduced compared with open surgery. Postoperative pain after LVHR is potentially associated with the type of mesh fixation, in particular with the use of transfascial sutures $[3,4,9,10]$. The type of mesh fixation also determines the amount of mesh shrinkage and thereby potentially determines the incidence of hernia recurrence.

Transfascial, nonabsorbable sutures offer initially greater tensile strength than spiral tacks [11, 12]. Experimental studies show no additional advantage in fixation strength when a distance smaller than $2 \mathrm{~cm}$ is used between individual sutures. Conversely, spiral tacks may be associated with intestinal erosion, fistulization, and intestinal adhesions with obstruction [13, 14].

This study was designed to compare mesh shrinkage and pain between transfascial sutures and spiral tacks for intraperitoneal fixation of meshes. The primary outcome parameter of this study was mesh shrinkage; secondary outcome parameters included postoperative pain and surgical complications.

\section{Patients and methods}

This report was prepared in accordance with the Consolidated Standards of Reporting Trials (CONSORT) statement [15]. 
Study design and protocol

The Ethics Committee of the University of Bern, Switzerland approved the study protocol. The design of the trial consisted of a pretreatment evaluation, randomized treatment with either suture or tack mesh fixation, in-hospital postoperative follow-up, and follow-up after discharge at 6 weeks and 6 months.

\section{Inclusion criteria}

All patients older than 18 years presenting with ventral hernia (incisional or spontaneous) with hernia diameter of $8 \mathrm{~cm}$ or less at its greatest diameter, and fit for surgery were consecutively enrolled in the study. Operations were performed in our clinic after written consent had been obtained.

\section{Exclusion criteria}

Patients with previous mesh implantation, body mass index (BMI) $>45 \mathrm{~kg} / \mathrm{m}^{2}$, pregnancy, contaminated abdominal cavity or long-term use of immunosuppressive agents were excluded from the study.

\section{Preoperative evaluation}

All patients received a complete physical examination and standard laboratory and radiological work-up prior to surgery. Anesthesiological risk was classified according to the American Society of Anesthesiologists (ASA) classification.

\section{Randomization}

Randomization in permutated blocks of 20 was performed using sealed envelopes. The random distribution between the two groups was assessed using www.randomization. com. Envelopes from patients who were excluded were discarded.

\section{Surgical technique}

Antibiotic prophylaxis with amoxicillin/clavulanic acid $1.2 \mathrm{~g}$ i.v. (Augmentin, GlaxoSmithKline, Münchenbuchsee, Switzerland) was given before the operation. Surgical technique of LVHR was performed as described previously [1]. Briefly, after establishing pneumoperitoneum $(12 \mathrm{mmHg}$ ), adhesions to the anterior wall surrounding the hernia were lysed, and the content of the hernia was reduced. The hernial sac was left in situ. After completion of adhesiolysis, pneumoperitoneum was released, the maximal hernia diameter was measured as well as the longitudinal and horizontal diameter, and an appropriatesized mesh was tailored to overlap the hernia margins by at least $5 \mathrm{~cm}$ on each side. Parietene ${ }^{\circledR}$ composite meshes (Sofradim Production: Covidien Group Trevoux, France) were used $[3,16,17]$. The margin of the mesh was marked with straight titanium clips every $5 \mathrm{~cm}$. These clips were applied from the lateral borders of the mesh to align the edge of the mesh precisely with the most lateral part of the clip.

For suture fixation, two different types of nonabsorbable monofilament sutures (Prolene ${ }^{\circledR}$ 0, and Ethilon ${ }^{\circledR} 0$; Ethicon Switzerland, Johnson \& Johnson Medical, CH-8957 Spreitenbach) were placed alternatively at $2-3 \mathrm{~cm}$ intervals along the mesh margin. The mesh was rolled up and inserted into the abdomen through a 12-mm trocar. After the mesh was positioned in the abdominal cavity, the suture ties were pulled through the abdominal wall using a suture passer and the threats were knotted smoothly with the knots buried in the subcutaneous tissue after reduction of the intraabdominal pressure to $8 \mathrm{mmHg}$. No drains were inserted, and fascial closure using sutures was performed at all 12-mm trocar sites. A compression dressing was applied over the hernial sac and removed before hospital discharge. In the group using spiral tacks for fixation, four nonabsorbable sutures were temporarily placed and passed through the abdominal wall to facilitate positioning of the mesh. Thereafter, the perimeter of the mesh was fixed to the posterior fascia at locations no more than $2 \mathrm{~cm}$ apart using the ProTak device (5-mm titan; AutoSuture Switzerland, $\mathrm{CH}-8832$ Wollerau). Finally, the sutures were removed.

\section{Postoperative management and follow-up}

The standard postoperative treatment was according to generally accepted principles. After discharge, patients were seen in our outpatient clinic after 6 weeks and after 6 months following hernia repair. After discharge, maximal load bearing of no more than $10 \mathrm{~kg}$ was recommended for 4 weeks. Follow-up after discharge included physical examination and routine laboratory tests in selected patients.

Pain management and assessment

All patients received standardized perioperative pain management according to established postoperative management protocols in our hospital. Patients were placed routinely on a patient-controlled analgesic device using standard settings for morphine administration or received peridural analgesia depending on the preoperative work-up and evaluation of the attending anesthetist. In addition, patients received paracetamol and opioids at a weightdependent dosage. After discharge, pain intensity was 
assessed at 6 weeks and 6 months using a visual analog scale (VAS) of $10 \mathrm{~cm}$. Moreover, pain frequency was recorded and categorized in five levels (from less than once a week to unbearable daily pain).

Assessment of mesh position and shrinkage

To detect mesh position and shrinkage, all patients underwent conventional abdominal X-ray examination in prone position, at day 2 postoperatively and after 6 weeks and 6 months. All X-ray images were digitally recorded. Analysis was done anonymously, performed by the same examiner throughout the trial. Titanium clips from the mesh margin were identified as reference points, and the X-ray area of the mesh was estimated using the planimetry tool of the IDS5 diagnostic imaging software (Sectra Imtec, Linköping, Sweden). The mesh surface area at 6 weeks and 6 months was compared with values determined 2 days postoperatively. All patients were imaged using similar specifications as previously published [18]. The source-toimage distance (SID), i.e., the distance from the tube to the image receptor, was $40 \mathrm{in}$. The kilovoltage peak $(\mathrm{kVp})$, i.e., the energy that penetrated the body, was between 70 and $75 \mathrm{kVp}$, depending on patient size. In the event that $\mathrm{X}$-ray image sizes were different between the three different time points, measurements were standardized by using the base of the third lumbar vertebral body as a scale of reference [18].

\section{Endpoints of the study}

The primary endpoint was mesh shrinkage. Secondary endpoints were postoperative pain, mesh dislocation, and surgical morbidity. All points of interest as well as all complications or adverse reactions were documented in the patient record form.

\section{Statistical analysis}

Statistical analysis was performed on an intention-to-treat basis. Since there were no prior data available documenting mesh shrinkage in humans in relation to the technique applied for mesh fixation, no power analysis was performed. Chi-square analyses were used to compare relationships among categorical variables, and the Fisher exact test was performed to compare proportions where appropriate. The Student $t$-test was used to compare means of quantitative variables. In case of nonnormal distribution, Mann-Whitney nonparametric analysis of variance was used for quantitative variables. All tests were performed bilaterally with 5\% significance threshold. All calculations were performed using SPSS (SPSS, Chicago, USA).

\section{Results}

The flowchart of participants through each stage of the trial is depicted in Fig. 1. Eligible patients were recruited between April 2005 and January 2008. After discharge, all participants were followed in our outpatient clinic at 6 weeks and 6 months after surgery.

Patient characteristics and surgical details

Baseline characteristics of patients are summarized in Table 1. No intraoperative complications occurred in either group. Operative details, surgical in-hospital morbidity, and follow-up details are presented in Table 2. There was no mesh dislocation in either group. Recurrent hernia was found in two patients (one in each group).

Postoperative mesh shrinkage

Representative examples of radiological follow-up from one patient are shown in Fig. 2. A significant decrease in horizontal mesh size was found after tack fixation (mean difference $-3.1 \pm 3.9 \%)$ versus fixation using sutures $(-0.1 \pm 2.3 \% ; p=0.018)$ (Fig. 3). Mean vertical mesh size was not significantly different between the two groups (tack fixation $-2.8 \pm 6.1 \%$, suture fixation $-0.7 \pm 4.1 \%$; $p=0.16)$. Mean mesh area in the tack fixation group was $-12 \%$ and in the suture fixation group $-2.9 \%$ at 6 months postoperatively when compared with postoperative day 2 $(p=0.061)$.

\section{Postoperative pain assessment}

Postoperative pain values are summarized in Table 3. A significantly higher pain score was observed in the suture fixation group at 6 weeks with minimal pain of VAS 2 compared with the tack fixation group $(p=0.008)$. After 6 months, no statistically significant differences in VAS score were seen between the two fixation methods.

\section{Discussion}

This study showed lower mesh shrinkage after suture fixation compared with tack fixation at 6 weeks and 6 months. The results from this study confirm experimental [11, 19] and clinical data [9] showing that tensile strength of suture fixation is significantly higher compared with tack fixation. A recent prospective study investigated the outcome between three different fixation methods: nonabsorbable suture, absorbable sutures, and double crown technique [9]. In that study postoperative pain was not significantly different between these groups. However, 


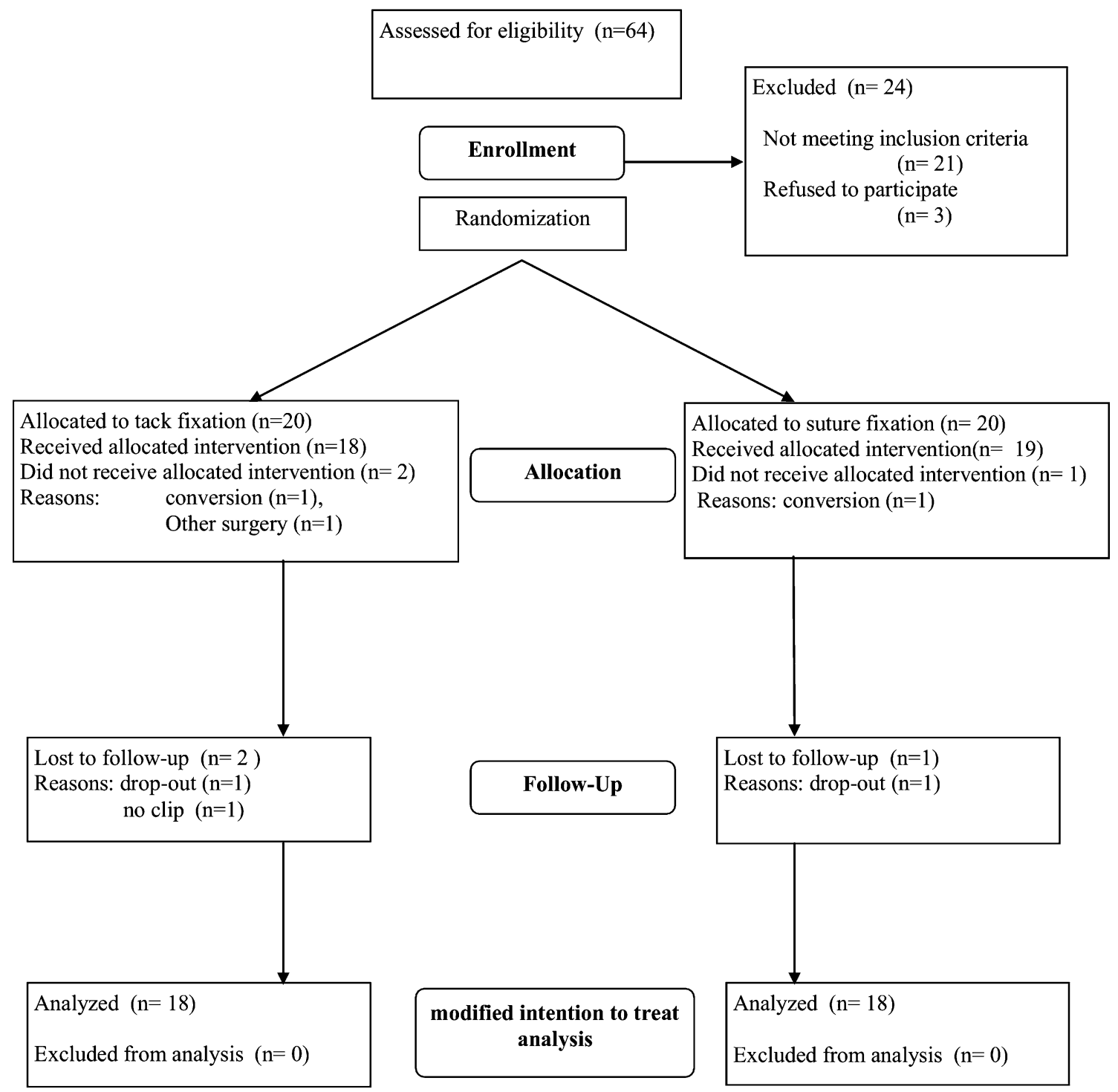

Fig. 1 Experimental flow chart

tacks were used in all three arms of the study, and only a mean of 8.8 sutures were applied in the suture groups. The present study adds further information by showing that mesh shrinkage is significantly increased by tack fixation.

No mesh displacement was observed in our study in either group despite more stable fixation to the abdominal wall using transfascial sutures. Thus, our results suggest that, for hernia size up to maximal diameter of $8 \mathrm{~cm}$, both fixation techniques seem to be sufficient to prevent early postoperative mesh dislocation.

Postoperative pain following suture fixation was significantly higher at 6 weeks postoperatively, and two patients suffered from nerve irritation at sites of sutures. However, after 6 months, no difference was seen between the two groups. Pain after mesh fixation with transfascial sutures is likely due to nerve irritation or entrapment and the relatively small distance between individual sutures used in our study. The significant reduction of pain between 6 weeks and 6 months post operation in these patients could be in response to desensitization of entrapped nerve fibers or in response to resolution of local inflammation. Therefore we recommend surgical revisions due to nerve irritation not earlier than 6 months postoperatively. For comparison of postoperative pain, only VAS scores $\geq 2$ were included in the analysis, because such values represent clinically relevant pain. Such assessment, however, may accentuate high pain values and underrate patients with low pain values.

A possible disadvantage of transfascial suture fixation is the prolonged operation time. This may be explained by the increased operative steps (fixation of the sutures on the mesh, elevation with suture passer, and knotting of sutures) 
Table 1 Baseline demographic and clinical characteristics of patients, modified intention-to-treat analysis $(n=34)$

\begin{tabular}{llll}
\hline Characteristics & Spiral tacks & Sutures & $p$-Value \\
\hline Sex ratio, M:F & $10: 6$ & $15: 3$ & 0.250 \\
Age (years) & $55(34-75)$ & $60(40-79)$ & 0.492 \\
BMI $\left(\mathrm{kg} / \mathrm{m}^{2}\right)$ & $28.7(24.2-35.4)$ & $28.4(23.6-35.9)$ & 0.870 \\
ASA $>2$ & $4 / 18(22 \%)$ & $8 / 18(38 \%)$ & 0.106 \\
Size of hernia $\left(\mathrm{cm}^{2}\right)$ & $12.6(1.0-56.0)$ & $12.6(3.1-168)$ & 0.982 \\
Site of hernia & & & \\
$\quad$ Umbilical & $7 / 18(39 \%)$ & $7 / 18(39 \%)$ & 0.807 \\
$\quad$ Epigastric & $6 / 18(33 \%)$ & $5 / 18(28 \%)$ & \\
$\quad$ Hypogastric & $1 / 18(6 \%)$ & $2 / 18(11 \%)$ & \\
$\quad$ Multiple & $2 / 18(11 \%)$ & $4 / 18(22 \%)$ & \\
Cause of hernia & & & \\
Incisional hernia & $11 / 18(61 \%)$ & $10 / 18(56 \%)$ & 0.497 \\
Primary hernia & $5 / 18(28 \%)$ & $8 / 18(44 \%)$ & \\
\hline
\end{tabular}

Quantitative data are given as median and range

ASA American Society of Anesthesiologists

Table 2 Operative details, morbidity, and follow-up, modified intention-to-treat analysis

\begin{tabular}{llll}
\hline Characteristics & Spiral tacks & Sutures & $p$-Value \\
\hline Mesh size $\left(\mathrm{cm}^{2}\right)$ & $263(113-600)$ & $300(113-750)$ & 0.236 \\
Operative time (min) & $92(45-310)$ & $120(75-240)$ & 0.039 \\
In-hospital stay (days) & $6(1-10)$ & $6(3-12)$ & 0.681 \\
Leave from work (days) & $35(4-120)$ & $21(0-105)$ & 0.440 \\
Late morbidity & $1 / 18(6 \%)$ & $3 / 18(17 \%)$ & 0.250 \\
Nerve irritation & $0 / 18$ & $2 / 18(11 \%)$ & \\
Mesh migration & 0 & 0 & \\
Recurrence & $1 / 18(6 \%)$ & $1 / 18(6 \%)$ & \\
\hline
\end{tabular}

Quantitative data are given as median and range necessary for mesh fixation. Depending on the size of the hernia and the implanted mesh size, the resulting higher costs due to prolonged operation time may at least partially compensate the higher costs for the stapling devices. Interestingly, we found more hematoma formation in the group with tack fixation, which may be due to manual counterpressure during tack application, or tack penetration into the abdominal wall itself.

Assessment of mesh shrinkage using serial conventional $\mathrm{X}$-ray imaging was safe and feasible. However, in the early postoperative period, X-ray imaging in prone position was associated with increased pain during examination. Another group used computed tomography (CT) scans for quantification of mesh shrinkage, which was possible because polytetrafluorethylene (PTFE)-based meshes were used [20]. Polypropylene-based meshes that were used in our study are not visible on CT scans. Furthermore, the results of our study are difficult to compare with those results, as different time points were used. The main advantage of conventional X-ray imaging is reduced exposure to radiation, allowing higher frequency of imaging during follow-up. Thus, with the examination performed in the present study, quantitative assessment of mesh shrinkage was possible. As shown, this test permits prospective studies for different mesh fixation devices with much smaller sample size.

One of the limitations of this study is the lack of a gold standard against which to compare the reliability and reproducibility of our method of mesh size assessment. Additionally, the impact of changes in body weight on radiological assessment has not been determined. The lack of such previous data of mesh shrinkage in patients made it impossible to perform a power calculation during the planning of the study.
Fig. 2 Example of radiologic assessment of mesh size and shrinkage. Plain radiographs are shown from one patient at day 2 (left panel) following surgery and after 6 months (right panel). The mesh was marked with titan clips every $5 \mathrm{~cm}$. Radiographs were taken from patients in supine position lying on the radiographic plate. Mesh size was estimated using planimetry software
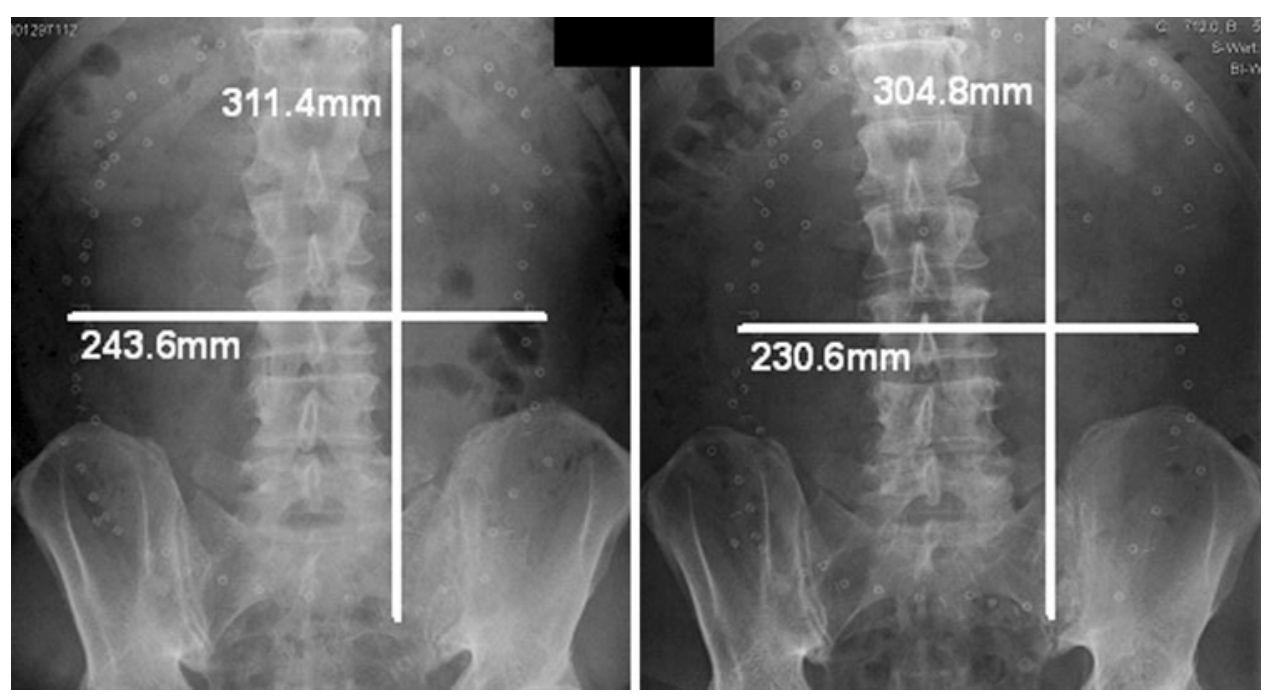

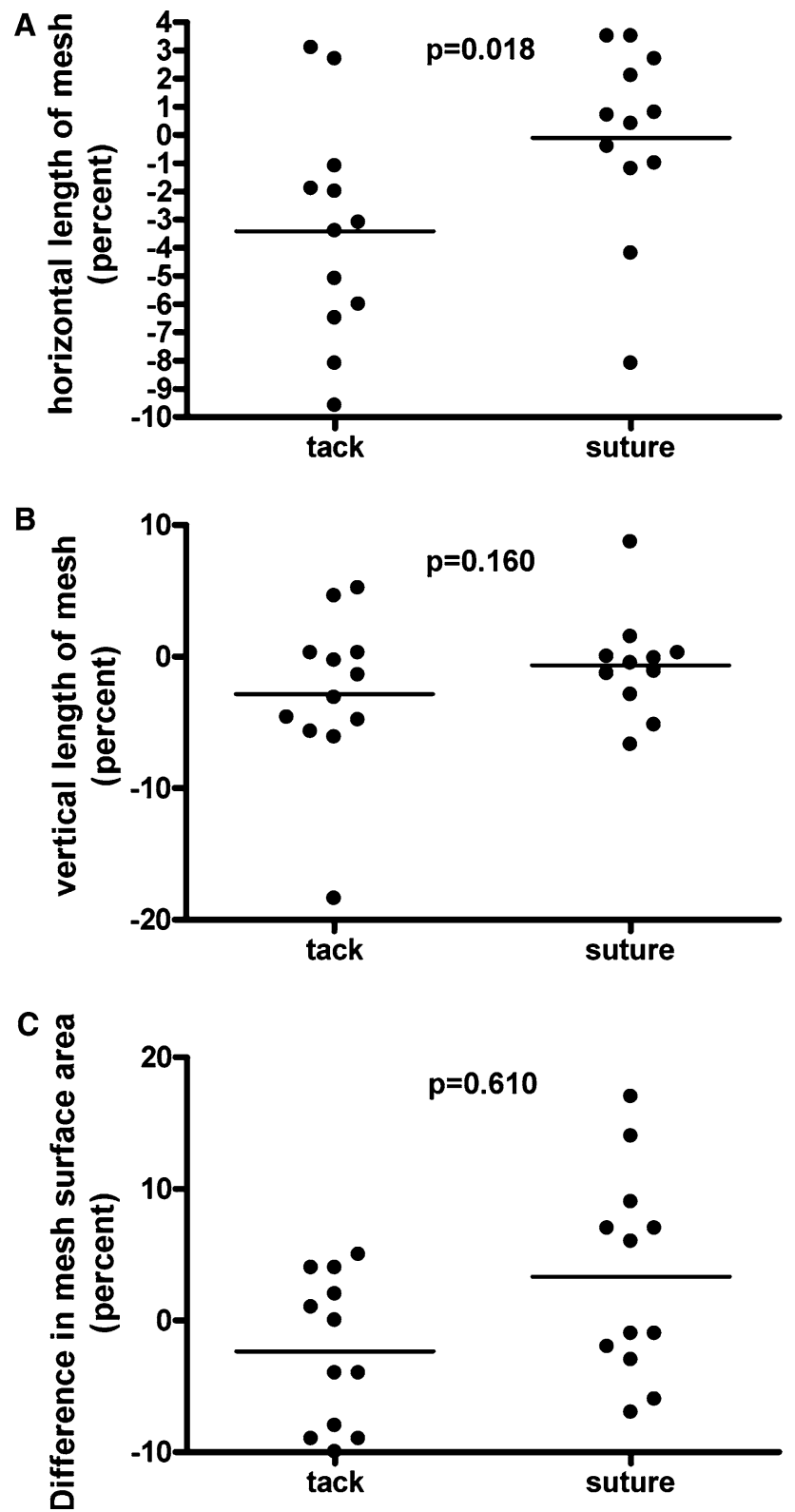

Fig. 3 Graphs representing change of mesh size at 6 month after mesh implantation in horizontal (A) and vertical (B) directions, and difference in calculated mesh area $(\mathbf{C})$

Table 3 Postoperative pain scores (VAS) after 6 weeks and after 6 months

\begin{tabular}{|c|c|c|c|}
\hline & Spiral tacks & Sutures & $p$-Value \\
\hline \multicolumn{4}{|c|}{ Total VAS score (mean \pm SD) } \\
\hline 6 weeks postoperatively & $2.5 \pm 0.8$ & $3.2 \pm 0.7$ & 0.345 \\
\hline 6 months postoperatively & $1.0 \pm 0.5$ & $0.7 \pm 0.3$ & 0.955 \\
\hline \multicolumn{4}{|l|}{$\mathrm{VAS} \geq 2(n)$} \\
\hline 6 weeks postoperatively & $28 \%(5 / 18)$ & $61 \%(11 / 18)$ & 0.020 \\
\hline 6 months postoperatively & $38 \%(7 / 18)$ & $50 \%(9 / 18)$ & 0.310 \\
\hline
\end{tabular}

$S D$ standard deviation
In conclusion, our study has demonstrated that both investigated types of mesh fixation are equally effective in preventing mesh shrinkage following repair for hernia smaller than $8 \mathrm{~cm}$. Sutures provide more rigid fixation to the fasciae of the abdominal wall, at the price of increased early postoperative pain. These findings are of importance for the treatment of large hernias that are typically exposed to more intraabdominal pressure requiring increased tensile strength for mesh fixation.

Acknowledgements The study was funded by Sofradim/Covidien. We thank Brigitte Wanner for meticulous data collection, control of data, and analysis of radiographs.

Disclosures Authors Guido Beldi, Markus Wagner, Lukas E. Bruegger, Anita Kurmann, and Daniel Candinas have no conflicts of interest or financial ties to disclose.

\section{References}

1. Beldi G, Ipaktchi R, Wagner M, Gloor B, Candinas D (2006) Laparoscopic ventral hernia repair is safe and cost effective. Surg Endosc 20:92-95

2. Bingener J, Buck L, Richards M, Michalek J, Schwesinger W, Sirinek K (2007) Long-term outcomes in laparoscopic vs open ventral hernia repair. Arch Surg 142:562-567

3. Chelala E, Thoma M, Tatete B, Lemye AC, Dessily M, Alle JL (2007) The suturing concept for laparoscopic mesh fixation in ventral and incisional hernia repair: mid-term analysis of 400 cases. Surg Endosc 21:391-395

4. Heniford BT, Park A, Ramshaw BJ, Voeller G (2003) Laparoscopic repair of ventral hernias: nine years' experience with 850 consecutive hernias. Ann Surg 238:391-399; discussion 399-400

5. Olmi S, Scaini A, Cesana GC, Erba L, Croce E (2007) Laparoscopic versus open incisional hernia repair: an open randomized controlled study. Surg Endosc 21:555-559

6. Kapischke M, Schulz T, Schipper T, Tensfeldt J, Caliebe A (2008) Open versus laparoscopic incisional hernia repair: something different from a meta-analysis. Surg Endosc 22:2251-2260

7. Asencio F, Aguilo J, Peiro S, Carbo J, Ferri R, Caro F, Ahmad M (2009) Open randomized clinical trial of laparoscopic versus open incisional hernia repair. Surg Endosc 23:1441-1448

8. Sajid MS, Bokhari SA, Mallick AS, Cheek E, Baig MK (2009) Laparoscopic versus open repair of incisional/ventral hernia: a meta-analysis. Am J Surg 197:64-72

9. Wassenaar E, Schoenmaeckers E, Raymakers J, van der Palen J, Rakic S (2010) Mesh-fixation method and pain and quality of life after laparoscopic ventral or incisional hernia repair: a randomized trial of three fixation techniques. Surg Endosc 24:1296-1302

10. Carbonell AM, Matthews BD, Kercher KW, Heniford BT (2003) Technique for introducing large composite mesh while performing laparoscopic incisional hernioplasty. Surg Endosc 17:1506; author reply 1507

11. van't Riet M, de Vos van Steenwijk PJ, Kleinrensink GJ, Steyerberg EW, Bonjer HJ (2002) Tensile strength of mesh fixation methods in laparoscopic incisional hernia repair. Surg Endosc 16:1713-1716

12. Winslow ER, Diaz S, Desai K, Meininger T, Soper NJ, Klingensmith ME (2004) Laparoscopic incisional hernia repair in a porcine model: what do transfixion sutures add? Surg Endosc $18: 529-35$ 
13. Karakousis CP, Volpe C, Tanski J, Colby ED, Winston J, Driscoll DL (1995) Use of a mesh for musculoaponeurotic defects of the abdominal wall in cancer surgery and the risk of bowel fistulas. J Am Coll Surg 181:11-16

14. Kaufman Z, Engelberg M, Zager M (1981) Fecal fistula: a late complication of Marlex mesh repair. Dis Colon Rectum 24: 543-544

15. Begg C, Cho M, Eastwood S, Horton R, Moher D, Olkin I, Pitkin R, Rennie D, Schulz KF, Simel D, Stroup DF (1996) Improving the quality of reporting of randomized controlled trials. The CONSORT statement. JAMA 276:637-639

16. McGinty JJ, Hogle NJ, McCarthy H, Fowler DL (2005) A comparative study of adhesion formation and abdominal wall ingrowth after laparoscopic ventral hernia repair in a porcine model using multiple types of mesh. Surg Endosc 19:786-790

17. Jacob BP, Hogle NJ, Durak E, Kim T, Fowler DL (2007) Tissue ingrowth and bowel adhesion formation in an animal comparative study: polypropylene versus proceed versus parietex composite. Surg Endosc 21:629-633

18. Sickle KR, Baghai M, Mattar SG, Bowers SP, Ramaswamy A, Swafford V, Smith CD, Ramshaw BJ (2005) What happens to the rectus abdominus fascia after laparoscopic ventral hernia repair? Hernia 9:358-362

19. Hollinsky C, Kolbe T, Walter I, Joachim A, Sandberg S, Koch T, Rülicke T, Tuchmann A (2010) Tensile strength and adhesion formation of mesh fixation systems used in laparoscopic incisional hernia repair. Surg Endosc 24:1318-1324

20. Schoenmaeckers EJ, van der Valk SB, van den Hout HW, Raymakers JF, Rakic S (2009) Computed tomographic measurements of mesh shrinkage after laparoscopic ventral incisional hernia repair with an expanded polytetrafluoroethylene mesh. Surg Endosc 23:1620-1623 